\title{
An improved photoelectric system for two-dimensional eye movement recording
}

\author{
C. R. BROWN and P. H. MOWFORTH \\ University of Sheffield, Sheffield S10 2TN, England
}

\begin{abstract}
Established photoelectric methods for measuring two-dimensional eye movements exist, but they suffer from a number of practical limitations, including inflexibility of the design, thermal drift, and difficulties in setting up and calibrating the system. In this paper, an improved design of transducer is described, together with a computerized method of calibration. Finally, some performance measurements are presented, showing the system to have an accuracy of a few minutes of arc.
\end{abstract}

The photoelectric technique for measuring eye movements by monitoring the motion of the boundary between the iris and the sclera, as first developed by Richter (1956), has seen many improvements, such as that described by Jones (1973) for measuring eye movements in two dimensions. The principle of Jones' method is illustrated in Figure 1. The eye is uniformly illuminated with light, and the amount of light reflected from two rectangular "receptive fields" positioned on the eye as shown is measured by two photodetectors. As the eye turns to the right (from the subject's viewpoint), more of the dark iris falls within the receptive field of the right-hand photodetector, so its output will decrease. Similarly, the output of the left-hand photodetector will increase. Thus for horizontal movements, the changes in the two photodetector outputs are in opposite directions. However, for vertical movements, the changes in the photodetector outputs are in the same direction. For example, if the eye turns downward, both photodetectors see more of the iris and so produce less output. Thus, signals representing horizontal and vertical eye movements may be obtained from the difference and the sum of the photodetector outputs, respectively.

Jones' (1973) transducer uses separate lenses to image the two receptive fields from the eye onto two photodetectors. The geometry of the receptive fields is determined by the size and shape of the sensitive area of the photodetectors and the demagnification introduced by the lens. Also, both lens/photodetector assemblies are fixed within a single plastic block. Thus, the size, shape, and relative positions of the receptive fields are rather firmly built into the apparatus, and uniformity of sensitivity over the area of each receptive field (which is assumed by the method) is at the mercy of the photodetectors. Also, there is no direct way of knowing where the receptive fields lie on the eye. Jones' transducer is mounted on an optician's trial spectacle frame. This arrangement, while convenient, prevents the subject's wearing his personal spectacles and, also, in our view, does not provide a sufficiently rigid mounting. Our

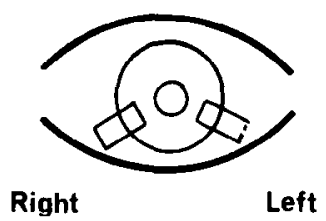

Figure 1. Positioning of the rectangular receptive fields on the eye. The larger circle represents the iris/sclera boundary when the subject is looking straight ahead.

device, while using the same principle as Jones' transducer, attempts to overcome these limitations.

\section{TRANSDUCER DESIGN}

The design of the modified transducer is shown in Figure 2. A single lens (f) of $22.4 \mathrm{~mm}$ diam and $20 \mathrm{~mm}$ focal length (Ealing Beck, Type 23-8774) forms an image of the eye at .5 magnification on a photographic mask (c). This mask is opaque, except for two transparent rectangular slits, each $2.3 \times 1.3 \mathrm{~mm}$. The mask is glued onto the front face of a cylindrical diffuser (a), $19 \mathrm{~mm}$ in diameter and $13 \mathrm{~mm}$ thick, machined from translucent Perspex. The two halves of the diffuser are separated by a thin aluminum plate (b) that prevents light's passing between the two halves. Light passing through the slits is diffused within the Perspex, and some of it falls upon two photodetectors ( $d$ and $e$ ) that fit into flat-bottomed holes drilled in the rear face of the diffuser. The photodetectors are large-area $(3 \times 3 \mathrm{~mm})$ silicon photodiodes mounted in TO-5 headers (Integrated Photomatrix, Ltd., Type IPL35).

The main purpose of the diffuser is to scatter the
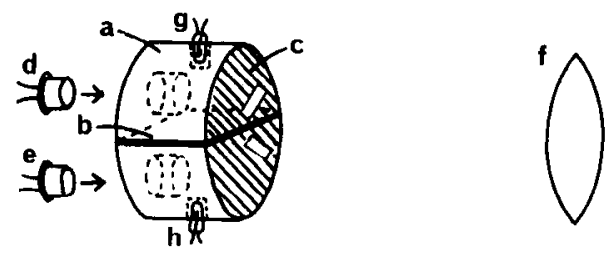

Figure 2. Schematic of the modified transducer. For explanation, see text. 


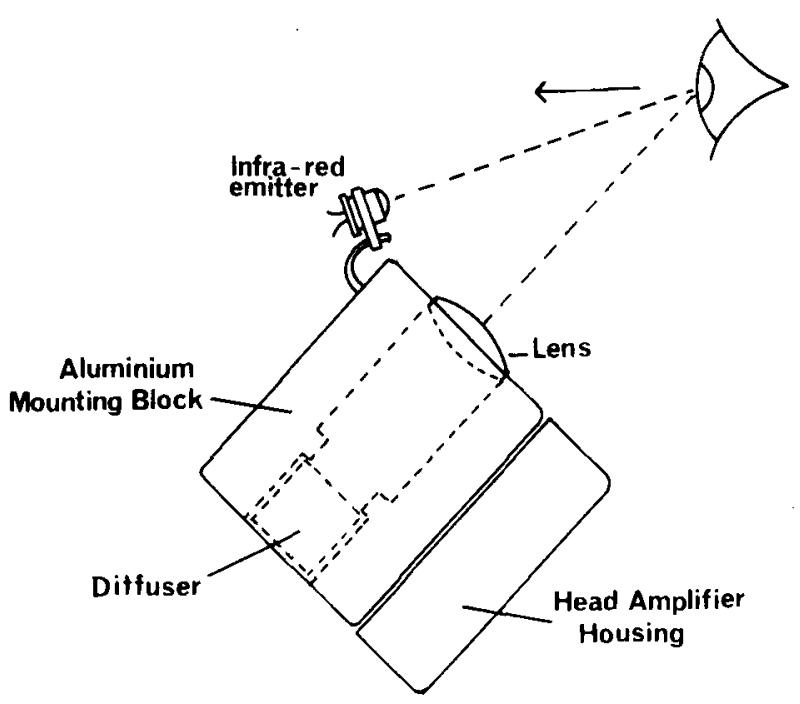

Figure 3. Transducer mounting. The lens and diffuser assembly are mounted in circular holes milled in the aluminum block. The block is mounted on an optical bench via two vernier sliders, allowing two-dimensional adjustment of position in a plane normal to the subject's line of sight. The optical axis of the system is inclined upward at $45 \mathrm{deg}$ to the horizontal.

light passing through the slits to ensure that the photodiodes are equally sensitive to light coming from all parts of the slit, that is, so that the sensitivity is uniform over the whole of the receptive field. However, the diffuser also provides an easy, reliable method of aligning the detector so that the receptive fields are correctly positioned on the eye. To do this, two subminiature filament lamps (Figure 2: $g$ and $h$ ) are fitted into two diametrically opposite holes drilled in the circumference of the diffuser. These flood the diffuser with light, thus back-illuminating the slits. Light emerging from the slits is back-projected by the lens onto the eye, forming two clearly visible patches of light that show exactly where the receptive fields lie. Of course, the filament lamps can only be used during alignment; they must be switched off while measurements are being taken.

The lens and diffuser assembly are mounted in an aluminum block $45 \mathrm{~mm}$ wide $\times 32 \mathrm{~mm}$ high $\times 50 \mathrm{~mm}$ deep (see Figure 3). Mounted on top of this on thick copper wire "stalks" are two infrared emitting diodes (Texas Instruments, Type TIL31). These produce a fairly narrow (10 deg) beam of light, so each emitter provides illumination for only one of the receptive fields. Each emitter is aligned manually to maximize the output of the corresponding photodiode. The emitters are connected in series and driven by a forward current of $130 \mathrm{~mA}$. Mounted below the aluminum block are a pair of head amplifiers, using a dual FET input opamp (RS Components, Type 305-939). These yield output voltages of the order of $500 \mathrm{mV}$.

\section{Advantages}

This design of transducer, while based on the same principle as that of Jones (1973), offers two important advantages. First, the use of a photographic mask to define the receptive fields makes it much easier to alter the size of the slits and their relative positions. It is not necessary to change the photodetectors, nor is one's choice constrained by the geometry of the detectors available. Also, one is free to choose photodetectors purely on the grounds of electrical suitability. Second, the back-projection alignment method provides a much easier and more reliable way of positioning the receptive fields on the eye than does Jones' alignment, which is, in his own words, "simply estimated." We have found that accurate alignment on the eye (particularly vertically) is most important for reliable two-dimensional measurements.

\section{Choice of Photodetector}

The choice of the IPL35 photodiode as the detector was made after testing a variety of detectors. The Clairex CL903 used by Jones (1973) is a cadmium selenide photoconductive type with a slow response time at low light levels and marked temperature dependence. Silicon planar phototransistors (e.g., the Motorola MRD300 or Texas Instruments TIL81) have fast response time and high sensitivity but produce drift problems due to the variation of current gain with temperature. Other types of silicon photodiodes were tried (e.g., the National Semiconductor NSL782), but the IPL35 gave the highest signal-to-noise ratio. The use of a photodiode, together with the light loss in the diffuser, results in very low photodetector currents (typically 50-100 nA). However, if FET input op-amps are used, this does not present a problem.

An additional advantage of a silicon detector is its relatively low sensitivity to visible light. This makes it possible to use the transducer with a fairly high intensity of ambient light from fluorescent (not tungsten) lamps. This not only makes operation more convenient, it also prevents the pupil's becoming too widely dilated. Too large a pupil causes problems if the receptive fields extend across the pupil boundary, because spurious signals are generated as the degree of dilation changes.

\section{Choice of Light Source}

Infrared emitting diodes have the advantage over filament lamps of being well matched with the spectral response of the silicon photodiodes. (Both peak around $800-900 \mathrm{~nm}$.) Consequently, there is no light "wasted," that is, serving only to heat the subject's eye. The TIL31, at a current of $130 \mathrm{~mA}$, was found to provide a fivefold increase in useful intensity compared with what could be achieved using a small filament lamp and infrared filter. However, no subjects have reported discomfort due to drying of the cornea. 


\section{Choice of Slit Size}

The size of the rectangular receptive fields used by Jones (1973) (measured on the eye) was $7.6 \times 1 \mathrm{~mm}$. We have found it advantageous both to shorten and to widen this rectangle, and we use dimensions of $4.6 \times 2.6 \mathrm{~mm}$. Shortening the rectangle reduces the risk of the receptive fields intersecting the lower lid or the pupil boundary. Widening the slit increases the sensitivity. Both modifications help to optimize the transducer for our application, in which a fairly small range ( $5 \mathrm{deg}$ ) of eye movements is of interest.

\section{Head Restraint}

Immobilization of the head with respect to the transducer is a crucial aspect of accurate eye movement measurements. For example, if the radius of the eyeball is taken to be $13 \mathrm{~mm}$, then a movement of the head (relative to the transducer) of only $.1 \mathrm{~mm}$ will be misinterpreted as an angular deviation of the eye of $26 \mathrm{~min}$ of arc (Carpenter, 1977, p. 314).

In the interests of comfort, we chose a combination of head restraint and bite bar. The head restraint comprises a crash helmet sawed in half and hinged at the back. Inside each half of the helmet is a polyethylene bag filled with expanded polystyrene granules. The helmet is closed over the subject's head and clamped at the front. This provides gross support and restraint for the head. Final immobilization is provided by a small bite bar, made from hard acrylic. This differs from normal in that it does not contain a full dental impression but locates on the front teeth only, an arrangement that we find comfortable and successful, given the head support and restraint provided by the helmet.

\section{CALIBRATION METHOD}

As has been explained, the principle of the method is that the outputs from the two photodetectors are subtracted and added in order to obtain orthogonal ( $\mathrm{x}$ and $\mathrm{y}$ ) measurements of eye position. In practice, however, this is oversimplified. It assumes equal sensitivities of the two detectors and symmetrical placement of the receptive fields on the eye. A more general and realistic statement is that horizontal and vertical components may be derived by taking suitable linear combinations of the photodetector outputs. Jones (1973) used an analog circuit to perform this operation; however, eight manual "gain" adjustments (four for each eye) must be made to ensure orthogonality, and correct sensitivity calibration, of the $\mathrm{x}$ and $\mathrm{y}$ outputs. This requires great care; errors in setting up will severely degrade the accuracy of the resolved $\mathrm{x}$ and $\mathrm{y}$ measurements.

If (as in our case) the transducers are used on-line to a computer, this calibration can be performed more easily and more accurately in software. The photodetector signals are therefore simply amplified and fed to four 10-bit analog-to-digital converters on the com- puter (two for each eye). The process of calibration for each eye amounts to deriving a linear transformation from the two photodetector outputs (A and B, say) to the coordinates of the point of regard ( $x$ and $y$ ). To do this, the subject looks in turn at four fixation points set in a square subtending 5 deg of visual angle. At each point, the program waits $500 \mathrm{msec}$ for the fixation to settle, and then it samples the photocell outputs for a further $500 \mathrm{msec}$. The individual samples are averaged to minimize errors due to miniature eye movements during the fixation. If the outputs fluctuate significantly during this period, due to a blink or a saccade, the program automatically repeats that fixation point. By taking the differences between appropriate pairs of readings (as shown in Figure 4), the program derives four quantities SAx, SAy, SBx, and SBy, representing the sensitivities of the $A$ and $B$ detectors to eye movements in $x$ and $y$. These values would allow us to predict what the $A$ and $B$ outputs would be for a given $x$ and $y$, as follows:

$$
\begin{aligned}
& A=(\operatorname{SAx} \cdot x)+(\text { SAy } \cdot y) \\
& B=(\operatorname{SBx} \cdot x)+(\operatorname{SBy} \cdot y)
\end{aligned}
$$

However, we actually require the inverse of this transformation, which is:

$$
\begin{aligned}
& \mathrm{x}=[(\mathrm{SBy} \cdot \mathrm{A}) / \mathrm{D}]-[(\mathrm{SAy} \cdot \mathrm{B}) / \mathrm{D}]+\mathrm{x} 0 \\
& \mathrm{y}=[(-\mathrm{SBx} \cdot \mathrm{A}) / \mathrm{D}]+[(\mathrm{SAx} \cdot \mathrm{B}) / \mathrm{D}]+\mathrm{y} 0
\end{aligned}
$$

where $\mathrm{D}=(\mathrm{SAx} \cdot \mathrm{SBy})-(\mathrm{SBx} \cdot \mathrm{SAy})$.

The zero-order, or "offset," terms $\mathrm{x} 0$ and $\mathrm{y} 0$ are calculated from a fifth fixation point at the center of the square. To minimize the effects of drift, this central fixation is presented both before and after the experi-

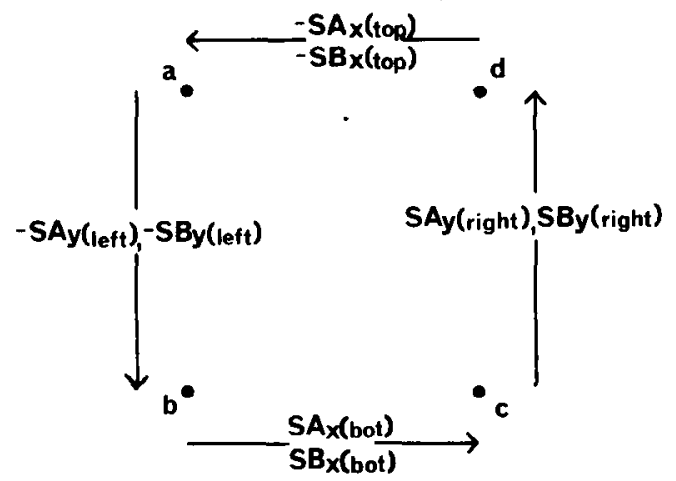

Figure 4. Fixation points used for calibration. The subject views the four points $a, b, c$, and $d$ in turn. The arrows indicate the eye movements and the sensitivities that are determined from each movement. Each sensitivity is derived from two movements, in opposite directions and on opposite sides of the square. In practice, each point is fixated four times (working around the square each time), so each sensitivity is an average of eight values derived from 16 fixations. The complete calibration takes about 20 sec. 


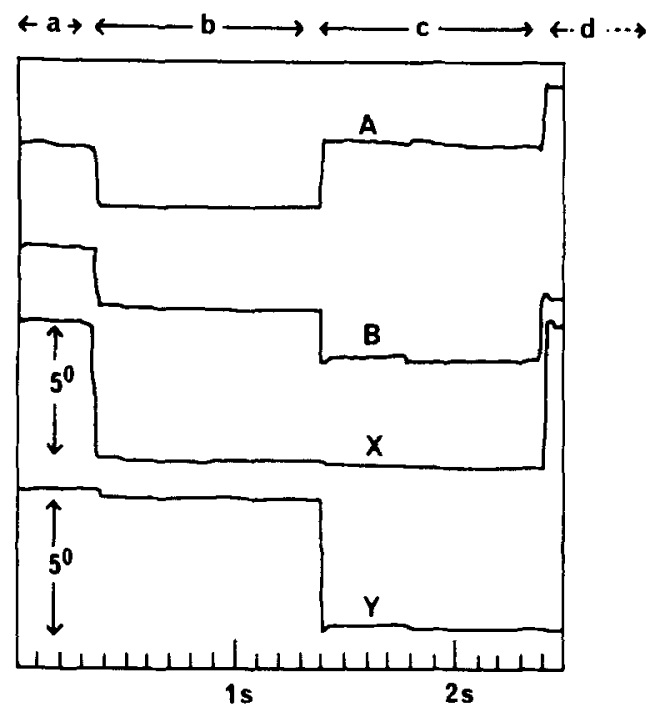

Figure 5. Signal waveforms obtained during calibration. A and $B$ are the outputs of the photodetectors; $X$ and $Y$ are the resolved horizontal and vertical components. Sections $a, b, c$, and $d$ of the waveform correspond to the four fixation points used (see Figure 4).

mental stimulus; $\mathrm{x} 0$ and $\mathrm{y} 0$ are averaged from these two measurements. An example of the actual waveforms obtained during calibration, together with the transformed $x$ and $y$ coordinates, is shown in Figure 5.

\section{PERFORMANCE}

The accuracy of an eye movement measurement system is very difficult to assess. The major problem is that while fixation points can be precisely positioned, there is no guarantee that the subject will fixate them accurately. For this reason, most workers have used artificial eyeballs to measure performance. While these facilitate measurement of, say, signal-to-noise ratio and thermal drift in the electronics, we do not consider them sufficient to establish the accuracy achieved when recording from a live subject.

One of the benefits of computer calibration is that the transducer sensitivities can be determined easily and with minimal error. Typical mean standard deviations of repeated measurements for horizontal and vertical sensitivity are about $7 \%$. Nonlinearity, which we define in the worst case as the percentage difference between sensitivity to movements on opposite sides of the calibration square [e.g., SAx(top) and SAx(bot) in Figure 4], is typically $9 \%$. Nonlinearity, however, is not a fixed property of the transducer. The values obtained were found to change in magnitude, and even in sign, if the transducer alignment was altered.

To measure cross talk between movements in $\mathrm{X}$ and $Y$, we asked subjects to follow a dot (size about $4.7 \mathrm{~min}$ ) through a movement of $56 \mathrm{~min}$ in a horizontal and then a vertical direction. The mean X "cross-talk" component during a vertical movement was about $5 \%$, and for the horizontal movement, the mean $Y$ "cross-talk" component was about $8 \%$ of the orthogonal change. However, the cross-talk signals were generally random, that is, not correlated with the step changes along the direction of the stimulus movement. It is therefore not appropriate to interpret these figures as a measure of systematic cross talk but rather merely as a manifestation of random movement along the orthogonal axis. (Systematic cross talk was measured using an artificial eyeball in which 2-deg horizontal and then vertical movements were made in order to ascertain what changes in measured position occurred in the orthogonal direction. For horizontal movements, the cross talk in $\mathrm{Y}$ was $3.3 \%$, and for vertical movements, the cross talk in $\mathrm{X}$ was $.2 \%$.)

As a final check on performance, subjects were asked to look at several fixation spots (size $=4.7 \mathrm{~min}$ ) within a 2.5 -deg square. Without discarding data, over 20 repeated measures the mean estimates of absolute position were within $4 \mathrm{~min}$ in both $\mathrm{X}$ and $\mathrm{Y}$ of the stimulus coordinates predicted from the calibration procedure. Standard deviations were about $9 \mathrm{~min}$ in $X$ and about $18 \mathrm{~min}$ in $\mathrm{Y}$. These results compare favorably with those of Carmody, Kundel, and Nodine (1980), who obtained mean calculated displacements of $30 \mathrm{~min}$ (on average) from the stimulus coordinates, using a similar computer calibration method, although with a different transducer system (Narco-Biosystems EyeTrack, Model 200-1).

Quantization error induced by the analog-to-digital converter is about $1 \mathrm{~min}$. Short-term drift (over a $2.5 \mathrm{-sec}$ period) is of the order of $1 \mathrm{~min}$. Over long time periods, drift can be readily compensated for by periodic recalibration.

The system has been used successfully for recording vergence responses from subjects looking at random dot stereograms. For examples of eye movements recorded on this system, see Mowforth, Mayhew, and Frisby (in press).

\section{Authors' Note Added to Proof}

Our deliberate choice of receptive field geometry restricts the operational field of view of our transducer to about $18 \mathrm{deg}$ horizontally by 9 deg vertically. Although we have not attempted any systematic assessment of accuracy outside the 5-deg square, experience suggests that our linear calibration transformation would not be adequate to maintain accuracy in the periphery of the field of view.

For a 5-deg field of view, we find that the calibration method will tolerate misalignment of up to $2 \mathrm{~mm}$ horizontally and $1 \mathrm{~mm}$ vertically without significant loss of accuracy. The constraints on horizontal alignment are that the receptive fields must straddle the limbus but must not overlap the pupil. For vertical alignment, the 
receptive fields must not overlap the lower lids, nor should they intersect the limbus too close to the horizontal diameter of the iris; otherwise, the y sensitivities drop considerably.

\section{REFERENCES}

Carmody, D. P., Kundel, L., \& Nodine, C. F. Performance of a computer system for recording eye fixations using limbus reflection. Behavior Research Methods \& Instrumentation, 1980, 12, 63-66.

CARpenter, R. H. S. Movements of the eyes. London: Pion, 1977.
Jones, R. Two dimensional eye movement recording using a photo-electric matrix method. Vision Research, 1973, 15, 425431.

Mowforth, P., Mayhew, J. E. W., \& Frisby, J. P. Vergence eye movements made in response to spatial frequency filtered random dot stereograms. Perception, in press.

Richter, H. R. Principes de la photo-electronystagmographie. Revue Neurologique, 1956, 96, 422-424.

(Received for publication November 6, 1980; accepted November 27, 1980.) 